\title{
Radiological examinations - Benefit vs radiation risk What we should know.
}

\author{
A Pallewatte MD \\ National Hospital of Sri Lanka
}

$\mathrm{X}$-rays are forms of radiant energy, like light or radio waves but unlike light, $x$-rays can penetrate the body forming an image of internal structures. The scientific measurement unit for radiation dose, known as effective dose, is the millisievert (mSv). Different tissues and organs have varying sensitivity to radiation exposure and therefore the radiation risk. Other radiation dose measurement units include rad, rem, Roentgen, Sievert, and Gray which are used for different purposes. For example, Plain chest $X$ ray gives an average dose of $0.1 \mathrm{mSv}$ while CT chest may give a dose of about $8 \mathrm{mSv}$.

Radiology and radiotherapy are not the only way for us to get radiation. There is background radiation from natural sources such as naturally occurring radioactive materials and cosmic radiation from outer space all the time.

As with other medical procedures, $x$-rays are safe when used with care. Radiologists and radiographers use the minimum amount of radiation necessary to obtain the needed results. Therefore properly conducted imaging carries minimal risks and should be performed when clinically indicated.

Therefore the referring clinician can decide on the benefit vs risk from the requested procedure with some background knowledge..

For low dose examinations, such as plain xrays this is generally an easy decision. For higher dose exams such as computed tomography (CT) scans and those involving the use of media it may need further evaluation. In children and in pregnancy it is vital to consider the indication and how the result of the examination helps in management. Other facts to consider are the number of previous radiation exposures the patient may have had and also the region to be examined. For example exposure of pelvis can be potentially harmful than an extremity.

While the vast majority of medical $x$-rays do not pose a critical risk to a developing fetus, the more mature the fetus, the lesser the risk. However Ultrasound which does not use $x$-rays may be more suitable in pregnancy and in children.

However, in nuclear medicine another precaution is advised for lactating women to avoid breastfeeding just after an examination.

In radiology the commonest examinations that can give higher dose are CT scans and interventional radiological procedures such as aneurysm coiling. Any examination may slightly increase the statistical risk cancer, but is vastly outweighed by the crucial benefit obtained by performing them.

Therefore this knowledge should not act as deterrent for using radiological examinations, but to get the maximum benefit for the patient.. In fact ultrasound and MRI use a different forms of energy which does not use ionizing radiation.

Author

- A Pallewatta Consultant Interventional Radiologist, National Hospital of Sri Lanka 